\title{
Multidrug resistance-associated protein 4 is a bile transporter of Clonorchis sinensis simulated by in silico docking
}

\author{
Fuhong Dai ${ }^{1 \dagger}$, Won Gi Yoo ${ }^{1 \dagger}$, Ji-Yun Lee ${ }^{1}$, Yanyan Lu', Jhang Ho Pak², Woon-Mok Sohn³ and Sung-Jong Hong ${ }^{1 *}$
}

\begin{abstract}
Background: Multidrug resistance-associated protein 4 (MRP4) is a member of the $C$ subfamily of the ABC family of ATP-binding cassette (ABC) transporters. MRP4 regulates ATP-dependent efflux of various organic anionic substrates and bile acids out of cells. Since Clonorchis sinensis lives in host's bile duct, accumulation of bile juice can be toxic to the worm's tissues and cells. Therefore, $C$. sinensis needs bile transporters to reduce accumulation of bile acids within its body.

Results: We cloned MRP4 (CsMRP4) from C. sinensis and obtained a CDNA encoding an open reading frame of 1469 amino acids. Phylogenetic analysis revealed that CSMRP4 belonged to the MRP/SUR/CFTR subfamily. A tertiary structure of CsMRP4 was generated by homology modeling based on multiple structures of MRP1 and Pglycoprotein. CSMRP4 had two membrane-spanning domains (MSD1 \& 2) and two nucleotide-binding domains (NBD1 \& 2) as common structural folds. Docking simulation with nine bile acids showed that CsMRP4 transports bile acids through the inner cavity. Moreover, it was found that CsMRP4 mRNA was more abundant in the metacercariae than in the adults. Mouse immune serum, generated against the CsMRP4-NBD1 (24.9 kDa) fragment, localized CSMRP4 mainly in mesenchymal tissues and oral and ventral suckers of the metacercariae and the adults.
\end{abstract}

Conclusions: Our findings shed new light on MRPs and their homologs and provide a platform for further structural and functional investigations on the bile transporters and parasites' survival.

Keywords: Clonorchis sinensis, Bile transporter, MRP4, ABCC4, Structure, Localization

\section{Background}

Clonorchiasis is a major endemic disease affecting over 35 million people in Asian countries including Korea, China, Thailand and Vietnam [1-3]. Clonorchis sinensis infections are caused by the ingestion of raw or undercooked freshwater fish that harbor the metacercariae [4]. Complications associated with the infection increase with an increase in the intensity and duration of the infection. Clonorchis sinensis has also been recognized by the World Health Organization as a biological carcinogen that can induce cholangiocarcinoma in humans [5].

C. sinensis migrates into the bile duct of the host and lives there. However, the bile duct can be regarded as an

\footnotetext{
* Correspondence: hongsj@cau.ac.kr

${ }^{\dagger}$ Equal contributors

'Department of Medical Environmental Biology, Chung-Ang University

College of Medicine, Seoul 06974, South Korea

Full list of author information is available at the end of the article
}

extreme environment, since accumulation of bile juice can be toxic to the worm's tissues and cells [6]. Among the various bile juice, lithocholic acid (LCA) has been proven to have a toxic effect on the survival of juvenile C. sinensis [7]. Therefore, it is important that the influx and efflux of bile acids should be balanced to prevent bile intoxication in the worm's body. In humans, there are many importers and exporters of bile juice circulation [8], such as apical sodium-dependent bile acid transporter (ASBT), $\mathrm{Na}^{+}$taurocholate co-transporting polypeptide (NTCP), Multidrug resistance protein (MRP), bile salts export pump (BSEP), and organic solute transporter (OST). Therefore, we believe that $C$. sinensis needs these bile transporters to reduce accumulation of bile acids within its body.

MRPs belong to a subfamily of the ATP binding cassette $(\mathrm{ABC})$ transporter family $[9,10]$. In higher animals, MRP4 is a unidirectional and distinctive bilaterally localized 
transporter in polarized cells, such as baso-lateral membrane of hepatocytes [11] and choroid plexus epithelial cells $[12,13]$. Such tissue-specific distribution suggests that MRP4 has multiple functions. MRP4 is responsible for pumping out a broad range of substrates, including bile acids, as well as for physiological regulation via transport of cyclic nucleotides out of cells [14].

Therefore in this study, we identified and characterized C. sinensis MRP4 (CsMRP4), the first MRP4 in trematodes, at the in silico, molecular, and biochemical levels. The structure of CsMRP4 was built using homology modeling, and the structural features and bile acid-binding affinities were investigated. CsMRP4 was found to be localized mainly in the mesenchymal tissues and oral suckers of $C$. sinensis adults and metacercariae.

\section{Methods}

Parasites and animals

Pungtungia herzi (Jinju, Korea), the second intermediate host of C. sinensis, was ground and digested as described by Dai et al. [15]. Metacercariae were then collected from the saline-rinsed digestive leavings under a dissecting microscope. Next, New Zealand white rabbits $(2.3 \mathrm{~kg}$; Koatech, Seoul, Korea) were infected with 200 metacercariae per rabbit twice in 1 week. Adult $C$. sinensis were then recovered from the rabbit livers after 2 months and stored in a $-80{ }^{\circ} \mathrm{C}$ freezer until use. Female 7 week-old BALB/c mice (Orient Bio Inc., Gyeonggi-do, Korea) were immunized with a bacterially-produced recombinant protein.

\section{CsMRP4 CDNA}

A putative MRP4 polypeptide sequence (GenBank ID: GAA49862.1) of $C$. sinensis was retrieved from the National Center for Biotechnology Information (NCBI) database. The coding DNA sequence (CDS) was obtained from the $C$. sinensis DNA scaffold (GenBank ID: DF142991.1) to which it belonged. DNA-walking was performed twice for CsMRP4-I and CsMRP4-II due to the long size (approximately $4 \mathrm{~kb}$ ) of the products. Two sets of PCR primers were designed according to CDS and synthesized (Bioneer, Daejeon, Korea) (Additional file 1: Table S1). Total cDNA of C. sinensis was prepared as described previously [15], and $50 \mathrm{ng}$ per reaction was used as the template for DNA-walking. PCR amplification was performed under the following conditions: pre-denaturation $\left(94^{\circ} \mathrm{C}\right.$ for $\left.5 \mathrm{~min}\right)$, amplification phase with 35 cycles $\left(94{ }^{\circ} \mathrm{C}\right.$ for $30 \mathrm{~s}, 55^{\circ} \mathrm{C}$ for $30 \mathrm{~s}, 72{ }^{\circ} \mathrm{C}$ for $2 \mathrm{~min} 15 \mathrm{~s}$ ), and final extension $\left(72{ }^{\circ} \mathrm{C}\right.$ for $10 \mathrm{~min}$ ). The PCR products were then purified using the QIAquick PCR purification kit (Qiagen, Hilden, Germany) and sequenced (Macrogen Inc., Seoul, Korea). The CsMRP4-I and CsMRP4-II sequences were used for assembling the putative CDS and translated into amino acid (aa) sequences. However, CsMRP4 was assumed to be incomplete at the $5^{\prime}$-end upon comparison with MRP4 of other species. Therefore, 5 '-rapid amplification of the cDNA ends (5'-RACE) was carried out to obtain the entire CDS. Total cDNA of $C$. sinensis was synthesized using the SMARTer ${ }^{\text {rm }}$ RACE cDNA amplification kit (Clontech, Mountain View, CA, USA) according to the manufacturer's instructions. The missing 5 '-end of CsMRP4 was amplified by RACE-PCR run using the 5'-RACE universal primer mix (UPM) and gene specific reverse primer (GSP). The PCR product was then confirmed using nested PCR, purified, and subjected to TOPO TA cloning (Invitrogen, Carlsbad, CA, USA). Through blue-white screening, the positive white colony was selected and reconfirmed by rapid colony PCR. Its plasmid DNA was extracted using the Plasmid Miniprep kit (Qiagen, Seoul, Korea) and sequenced (Macrogen Inc., Seoul, Korea). The primers used for RACE-PCR, DNA-walking, and multiple sequencing are listed in Additional file 1: Table S1.

\section{In silico methods for characterizing sequence features}

For CsMRP4, the isoelectric point (pI) and molecular weight $(\mathrm{Mr})$ was estimated using the ExPASy ProtParam Tool (http://web.expasy.org/protparam/). CsMRP4 was blasted against UniProtKB/Swiss-Prot v. 2017_07 [16]. Domain organization and residue annotation were conducted using the Conserved Domain Database (CDD) [17] and InterProScan v. 64 [18].

\section{Phylogenetic analysis}

In order to confirm that CsMRP4 belongs to a MRP subfamily and to infer its phylogenetic relationship with the $\mathrm{ABCC}$ and $\mathrm{ABCB}$ subfamilies, 12 canonical $\mathrm{ABCC}$ proteins and 11 canonical $A B C B$ proteins were retrieved from UniProtKB/Swiss-Prot v. 2017_07 [16]. Multiple sequence alignment was performed using the L-INS-i method of MAFFT v. 7.299 [19]. An evolutionary history was inferred by employing the neighbor-joining (NJ) method using MEGA v. 6.06 [20]. All the positions containing gaps and missing data were eliminated.

\section{Homology modeling and refinement}

The standard protocol of YASARA Structure v. 17.6.5 [21] was used to build the homology models of CsMRP4. To obtain these models, PSI-BLAST [22] was carried out against PDB entries (updated August, 2017) [23]. After building the homology models for each template, the models were submitted to high-resolution energy minimization using a YASARA force field [24]. The result was then validated to ensure that the refinement did not move the model in the wrong direction. Finally, a hybrid homology model was obtained by combining the best scoring parts of the four models. In addition, 
potential errors in the 3D models were evaluated using a Ramachandran plot [25] and ERRAT [26].

\section{Structure-based function analysis}

Structural conservation was calculated and visualized using ENDscript/ESPript v. 3.0 [27] with the PDBAA95 database, E-value of $1 \mathrm{e}-12$, and contact range of $2.7 \AA$. $\mathrm{COACH}$ [28] was used to predict ligand-binding sites in CsMRP4. SDF files for bile acids were retrieved from the PubChem database [29] as of August 2017 and transformed into the MOL2 format using OpenBabel [30]. Bile acids were docked into CsMRP4 using PyRx v. 0.8 [31], which includes AutoGrid [32] and AutoDock Vina [33]. A grid box extended to all membrane-spanning domains (MSDs) of CsMRP4; no information regarding the exact location of the binding sites of the various bile acids was available. Active site dimensions were set as the grid size of center X: $25.6 \AA$, center Y: $-13.5 \AA$, and center Z: $-6.5 \AA$, and 8 maximum exhaustiveness was calculated for each bile acid. All structure visualizations were carried out using UCSF CHIMERA v. 1.10.2 [34] and PyMOL Molecular Graphics System v. 1.7.4.5 (Schrödinger, LLC., New York, NY, USA).

\section{Quantitative measurement of CsMRP4 developmental expression}

To evaluate the mRNA expression level in different developmental stages, quantitative real time PCR (Q-rt.PCR) was performed. Primers were designed using Oligo-primer analysis software v. 6.71 (Molecular Biology Insights, Cascade, WA, USA) (Additional file 1: Table S1). Calcyphosine (CAP) and phosphoglycerate kinase (PGK) were employed as the reference genes [35]. Q-rt.-PCR reaction mixtures were prepared in triplicate using LightCycler FastStart DNA Master SYBR Green I Kit (Roche, Mannheim, Germany), with each reaction containing $50 \mathrm{ng}$ of total cDNA of the adults or metacercariae. Q-rt.-PCR was performed on LightCycler 2.0 (Roche, Penzberg, Germany) with the following thermal cycle parameters: pre-heating $\left(95^{\circ} \mathrm{C}\right.$ for $15 \mathrm{~min}$ ), $40 \mathrm{cy}-$ cles of $95{ }^{\circ} \mathrm{C}$ for $10 \mathrm{~s}, 48{ }^{\circ} \mathrm{C}$ for $10 \mathrm{~s}$, and $72{ }^{\circ} \mathrm{C}$ for $30 \mathrm{~s}$. The relative transcription ratio was calculated according to the $2^{-\Delta \Delta \mathrm{Ct}}$ method [36].

\section{Production of recombinant protein}

A cDNA fragment encoding CsMRP4-NBD1 was amplified using PCR (Additional file 1: Table S1). The purified PCR product was then subcloned into pET23b and confirmed by colony PCR and restriction enzyme digestion. Plasmid DNA of the positive clone was extracted using the QIAprep Spin Miniprep kit (Qiagen, Hilden, Germany) and sequenced (Macrogen, Seoul, Korea). The correct construct was then transformed in Escherichia coli BL21[DE3]pLysS (Novagen, San Diego, CA, USA) by heat-shock at $42{ }^{\circ} \mathrm{C}$ for $30 \mathrm{~s}$ and spread on LB/ampicillin/chloramphenicol agar. After overnight incubation at $37{ }^{\circ} \mathrm{C}$, a single colony was picked from the LB plate and grown in $\mathrm{LB} /$ ampicillin liquid medium by shaking vigorously at $37{ }^{\circ} \mathrm{C}$. The recombinant(r) CsMRP4-NBD1 was then induced with $0.1 \mathrm{mM}$ isopropyl- $\beta$ - $D$-thiogalactopyranoside (IPTG) (TaKaRa, Shiga, Japan) for $5 \mathrm{~h}$. The bacteria were then harvested, and recombinant protein was purified as described previously [15].

\section{Production of mouse immune serum}

The rCsMRP4-NBD1 was separated by $12 \%$ sodium dodecyl sulfate polyacrylamide gel electrophoresis (SDS-PAGE) and cut off alone to obtain specific antigens for mouse immunization. The gel slice was equilibrated and homogenized in pre-cooled $1 \times$ PBS by complete grinding. The liquid homogenate containing rCsMRP4-NBD1 was then injected into $\mathrm{BALB} / \mathrm{c}$ mice according to an immunization method [37]. Blood was drawn from the eye and stored at room temperature for 1 to $2 \mathrm{~h}$. The immune serum was obtained by centrifugation at $4000 \times \mathrm{rpm}$ for $20 \mathrm{~min}$. In order to examine the antibody titer in the immune serum against rCsMRP4-NBD1 and native CsMRP4, C. sinensis crude extracts were prepared using the Mem-PER Plus Membrane Protein Extraction Kit (Thermo scientific, Rockford, USA) following the manufacturer's instructions. The crude antigen was then examined to determine its concentration using the Bio-Rad protein assay (BioRad, Hercules, CA, USA) and then stored as aliquots at $-70{ }^{\circ} \mathrm{C}$ until use.

The reactivity of the antibody was checked by western blot against recombinant protein and immuno-enhanced chemiluminescence (ECL) against native CsMRP4 in crude extracts. The rCsMRP4-NBD1 and $C$. sinensis crude antigens were loaded onto SDS gels for electrophoresis and then transferred to nitrocellulose membranes (GE Healthcare Life Sciences, Seoul, Korea). The membranes were then blocked using $5 \%$ skim milk in PBS/0.05\% Tween20, followed by incubation with mouse immune serum at 1:400 at $4{ }^{\circ} \mathrm{C}$ overnight and then with goat-anti-mouse-IgG alkaline phosphatase-conjugated antibody (Sigma-Aldrich, St. Louis, MO, USA) at 1:5000 for western blotting or with peroxidase-conjugated AffiniPure Goat Anti-Mouse IgG antibody (Jackson ImmunoResearch Inc., West Grove, PA, USA) at 1:10,000 for ECL at room temperature for $2 \mathrm{~h}$. Normal mouse serum was used as the negative control. The recombinant protein was visualized by color developing in BCIP/NBT (Sigma-Aldrich, St. Louis, MO, USA). The native CsMRP4 was detected using a ECL solution kit (Bio Sesang, Seoul, Korea) and visualized using ImageQuant LAS 4000 (GE Healthcare Bio-Sciences, Amersham, UK). 


\section{Immunohistochemical staining}

Paraffin block preparation and immunohistochemical staining were performed using our previously described methods [15]. Mouse anti-NBD1 immune serum diluted at 1:200 served as the primary antibody. Normal mouse serum was used as the negative control. Dako EnVision + System-horseradish peroxidase-labeled polymer anti-mouse IgG (Dako Cytomation, Glostrup, Denmark) diluted at 1:400 was used as the secondary antibody.

\section{Results and discussion}

\section{Identification and molecular characteristics}

The complete coding cDNA sequence (4410 nt) of CsMRP4 was obtained through DNA-walking and 5'-RACE on the C. sinensis total cDNA. Its open reading frame was 1469 aa in length (Additional file 2: Figure S1 and Additional file 3: Figure S2). The Mr. of CsMRP4 was about $165.5 \mathrm{kDa}$ and its $\mathrm{pI}$ was estimated to be 6.5. BLASTP was performed against the UniProtKB/Swiss-Prot database [16], which is a high-quality, manually annotated, and non-redundant protein database. Annotation information from NCBI nonredundant and UniProtKB/TrEMBL databases need to be further reviewed, since those contents were generated using in silico annotation or large-scale functional prediction. CsMRP4 is the closest to human MRP4 (HsMRP4) (UniProt ID: O15439) with an E-value of 5.3e-147 and identity of 44.2\%, followed by HsMRP6 (UniProt ID: O95255) of 8.8e-89 and 38.4\%, Mus musculus MRP3 (MmMRP3) (UniProt ID: B2RX12) of $5.1 \mathrm{e}-104$ and $38.2 \%$, and MmMRP5 (UniProt ID: Q9R1X5) of 2.9e-115 and $38.0 \%$. CsMRP4 was significantly matched to multiple MRPs since the highest-scoring pairwise alignment was found predominantly in the NBD2 region, which is particularly conserved among $\mathrm{ABC}$ family transporters [38]. As a subfamily of the $A B C$ family of transporters, the MRP subfamily contains 12 members including MRP1-9, cystic fibrosis transmembrane conductance regulator (CFTR), sulfonylurea receptor 1 (SUR1), and SUR2 [9, 10]. The highest identity (39.0\%) was observed between CsMRP4 and HsMRP4 in comparison with canonical human MRP/SUR/CFTR subfamily (Additional file 4: Table S2). Thus, this clone was designated as CsMRP4.

\section{MRP-specific functional domains}

Functional domains in CsMRP4 revealed diverse characteristics of $\mathrm{ABC}$ transporters. There were "ABC transporter type 1, transmembrane domain" (InterProScan ID: IPR011527) in two regions, aa64-378 and aa831-1162. Two domains, MSD1 and MSD2, consisted of six transmembrane $\alpha$-helices each. As intracellular NBDs that linked with the MSDs, "ABCC_MRP_domain1" (CDD ID: cd03250) for NBD1 and "ABCC_MRP_domain2" (CDD ID: cd03244) for NBD2 were found in the aa407-603 and aa1174-1394 regions, respectively. CsMRP4 had a single four-domain organization of MSD1-NBD1-MSD2-NBD2, which is common to all short forms of the ABCC subfamily, such as MRP4, 5, 8, 9, and CFTR [14].

Like in typical MRPs, there were several conserved motifs in the NBD1 of CsMRP4, such as an ATPbinding site $\left({ }^{441} \mathrm{GCxKSSx}_{26} \mathrm{Qx}_{78} \mathrm{DDx}_{31} \mathrm{~N}^{585}\right)$, ABC transporter signature motif $\left({ }^{528}\right.$ LSGGQKARIG $\left.^{537}\right)$, Walker A/ P-loop $\left({ }^{438}\right.$ GPVGCGKS $\left.{ }^{445}\right)$, Walker B $\left({ }^{548}\right.$ FLLLDD $\left.^{553}\right)$, D-loop $\left({ }^{556} \mathrm{AAVD}^{559}\right)$, Q-loop/lid $\left({ }^{470} \mathrm{YMPQ}^{473}\right)$, and $\mathrm{H}$ loop/switch region $\left({ }^{581}\right.$ LLVTNQL $\left.^{587}\right)$. These motifs play pivotal roles in transporting substrates via conformational changes between outward-facing and inwardfacing forms. Dimerization of two NBDs forms the nucleotide-binding site between the Walker A/P-loop, Walker B, and ABC signature motif. The bound ATP is hydrolyzed to provide energy in order to efflux endogenous and xenobiotic substrates from cells to the extracellular milieu [39].

\section{Phylogenetic inference}

An NJ method-based phylogenetic inference verified that CsMRP4 belongs to the MRP/SUR/CFTR subfamily by comparing it with the 12 members of subfamily $\mathrm{C}$ and 11 members of subfamily $B$ of the $A B C$ family (Fig. 1). Members of the ABCB subfamily were clearly out-grouped as one cluster despite the close similarity in terms of both sequence and structure between MRP and P-glycoprotein (P-gp) of ABCB members [39]. Moreover, CsMRP7 (GenBank ID: AOE23877.1), which was annotated using the same approach, was grouped with HsMRP7.

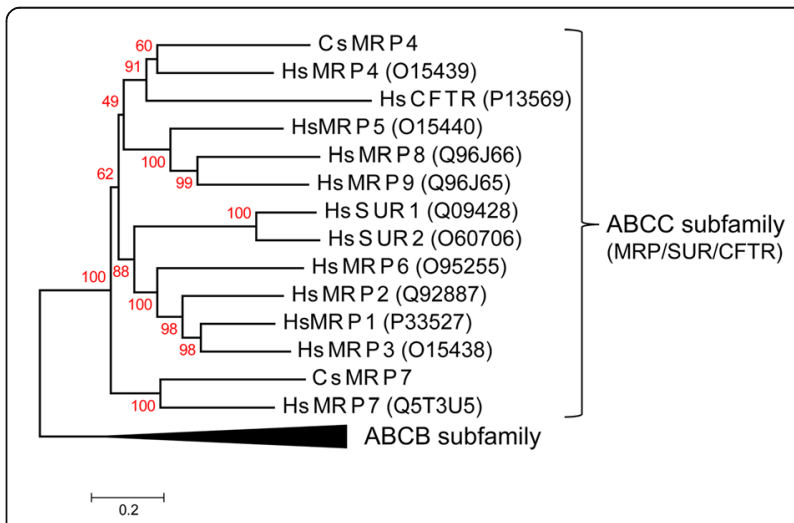

Fig. 1 Phylogenetic relationship between CsMRP4 and members of the MRP/SUR/CFTR subfamily. Bootstrap values (1000 replicates) are shown next to the branches. Scale bar represents amino acid substitutions. UniProtKB/Swiss-Prot IDs for the ABCC subfamily are shown in parentheses. IDs for the ABCB subfamily are P-gp1 (P08183), ATP1 (Q03518), ATP2 (Q03519), P-gp3 (P21439), ABCB5 (Q2M3G0), MT-ABC3 (Q9NP58), ABC7 (O75027), M-ABC1 (Q9NUT2), TAPL (Q9NP78), M-ABC2 (Q9NRK6), and BSEP (O95342) 


\section{D homology model of CsMRP4}

Several MRP4-related PDB structures were found from the PSI-BLAST search with experimentally characterized PDB structures, although there is no solved PDB structure of MRP4 elucidated so far. YASARA Structure selected the templates, such as Bos taurus MRP1 (PDB ID: 5UJA) with $35.2 \%$ identity, Caenorhabditis elegans P-gp (PDB ID: 4F4C) with 19.1\% identity, MmMRP1 (PDB ID: $4 \mathrm{M} 1 \mathrm{M}$ ) with $18.9 \%$ identity, and MmMRP1 (PDB ID: 4Q9H) with $18.3 \%$ identity. Each homology model, built with each template, was refined by unrestrained high-resolution energy minimization using the latest knowledge-based YASARA force field [24]. As a final model, a hybrid homology model was assembled by combining the best scoring parts of the four models and then refined with energy minimization. Out of 1469 residues, 1425 residues were modeled, omitting 44 C-terminal residues, since YASARA Structure does not perform ab initio or threading modeling (Fig. 2).

The final model proved highly accurate based on the following validation. The overall Z-score of the resulting hybrid model was -1.4 using internal quality evaluation of YASARA Structure. A Z-score indicates the number of standard deviations the model quality is away from the average high-resolution X-ray structure. Moreover, a
Ramachandran plot [40] of the final model showed that $90.7 \%$ of all the residues were found in the most favored regions, $8.4 \%$ in additional allowed regions, and only $0.3 \%$ in disallowed regions (Additional file 5: Figure S3). These results indicated that the backbone dihedral angles were highly accurate. The ERRAT value, as an overall quality score, was $98.4 \%$ (Additional file 6: Figure S4). The final model of the CsMRP4 in PDB format can be found in Additional file 7 .

The 3D structure formed MSD1-NBD1-MSD2-NBD2 as a common structural fold of $\mathrm{ABC}$ transporters (Fig. 2a). MSD1 and MSD2 were made up of TM1-6 and TM7-12, respectively (Fig. 2b). When CsMRP4 was compared structurally with the 38 homologs from PDB entries using ENDscript [27] with strict parameters, two NBDs were found to be highly conserved and two MSDs were less conserved (Fig. 2c). Furthermore, NBD2 of CsMRP4 was significantly more conserved than NBD1. At the sequence level, even though CsMRP4 was compared with five short forms of the ABCC subfamily, NBD2 contained 56 identical residues but NBD1 had only 41 identical residues (Fig. 3a, b). These results corroborate previous findings showing that NBD2 of CsMRP7 is more conserved than NBD1 at the structural level [15].

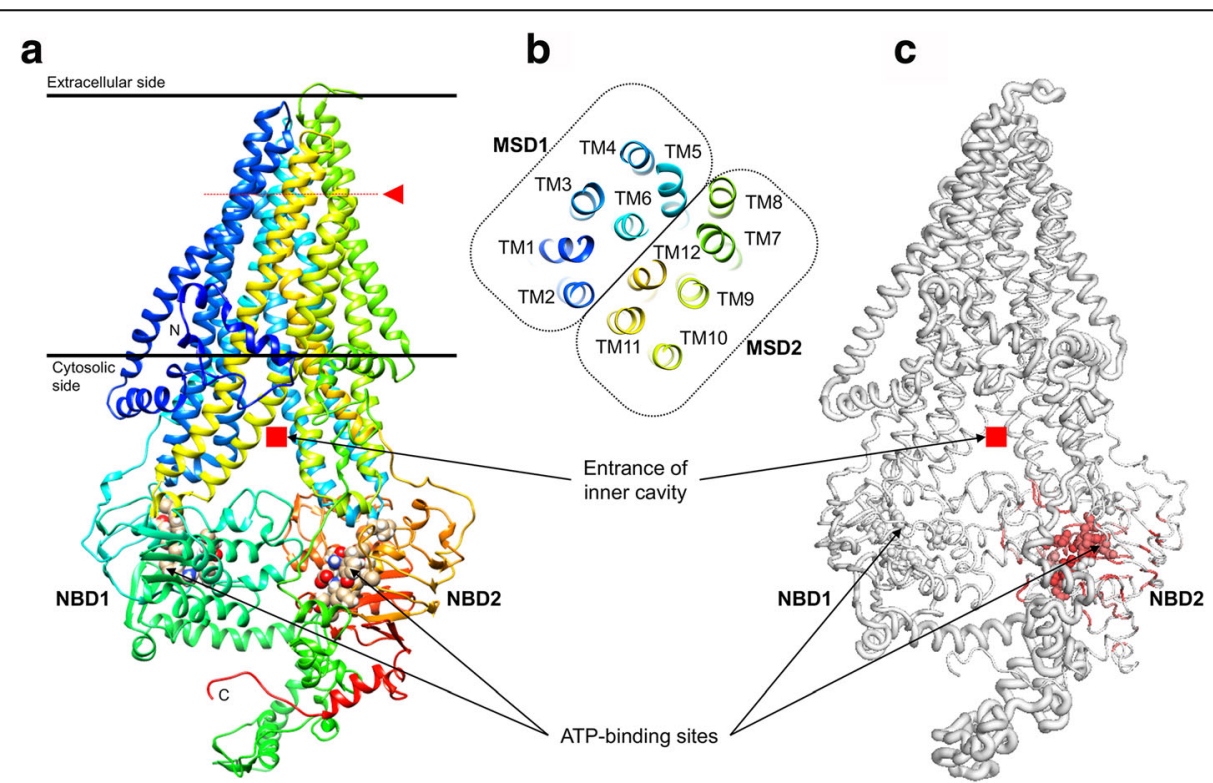

Fig. 2 Structural characterization and conservation of CSMRP4. a A 3D homology model was built based on the solved structure templates. The a-helix and $\beta$-strand are depicted as ribbon diagrams, and coiled-coil is depicted as a line. TM a-helices are colored and numbered from the N-terminus (blue) to the C-terminus (red). The red rectangles indicate the entrance of inner cavity, and spheres correspond to residues coordinating ATP-binding sites. $\mathbf{b}$ TM a-helices, as viewed perpendicular to the horizontal plane marked with red arrowheads. c The degree of sequence conservation is colored using a color gradient from white (divergent) to red (conserved). Structural conservation corresponds to the radii of the backbone sausage representation, which is proportional to the root-mean-squared deviation at each position between structure alignments. PDB IDs of the identified homologs are as follows: 5W81_A, 5UAK_A, 5UJ9_A, 4C3Z_A, 2PZG_A, 3GD7_A, 4Q4J_B, 1R0Z_A, 2HYD_A, 4Q7M_B, 4Q4J_A, 5DGX_A, 5IDV_A, 4MYC_A, 3WMF_A, 5MKK_A, 1MV5_A, 4MRN_A, 3NH6_A, 2FFB_A, 5EUM_A, 4F4C_A, 2GHI_A, 5MKK_B, 4Q9H_A, 4AYW_A, 3VX4_A, 5U1D_B, 5U1D_A, $4 \mathrm{PLO} \_\mathrm{A}, 5 \mathrm{~L} 22 \_\mathrm{B}, 4 \mathrm{U} 00 \_\mathrm{A}, 4 \mathrm{~K} 8 \mathrm{O} \_\mathrm{A}, 4 \mathrm{MKI} \_\mathrm{B}, 4 \mathrm{HUQ} \mathrm{B}, 3 \mathrm{TUJ} \_\mathrm{C}, 5 \mathrm{NIK} \_$J, and 5JSZ_A. Structural alignment and image rendering were carried out using ENDscript and PyMOL (See details in the Methods section) 


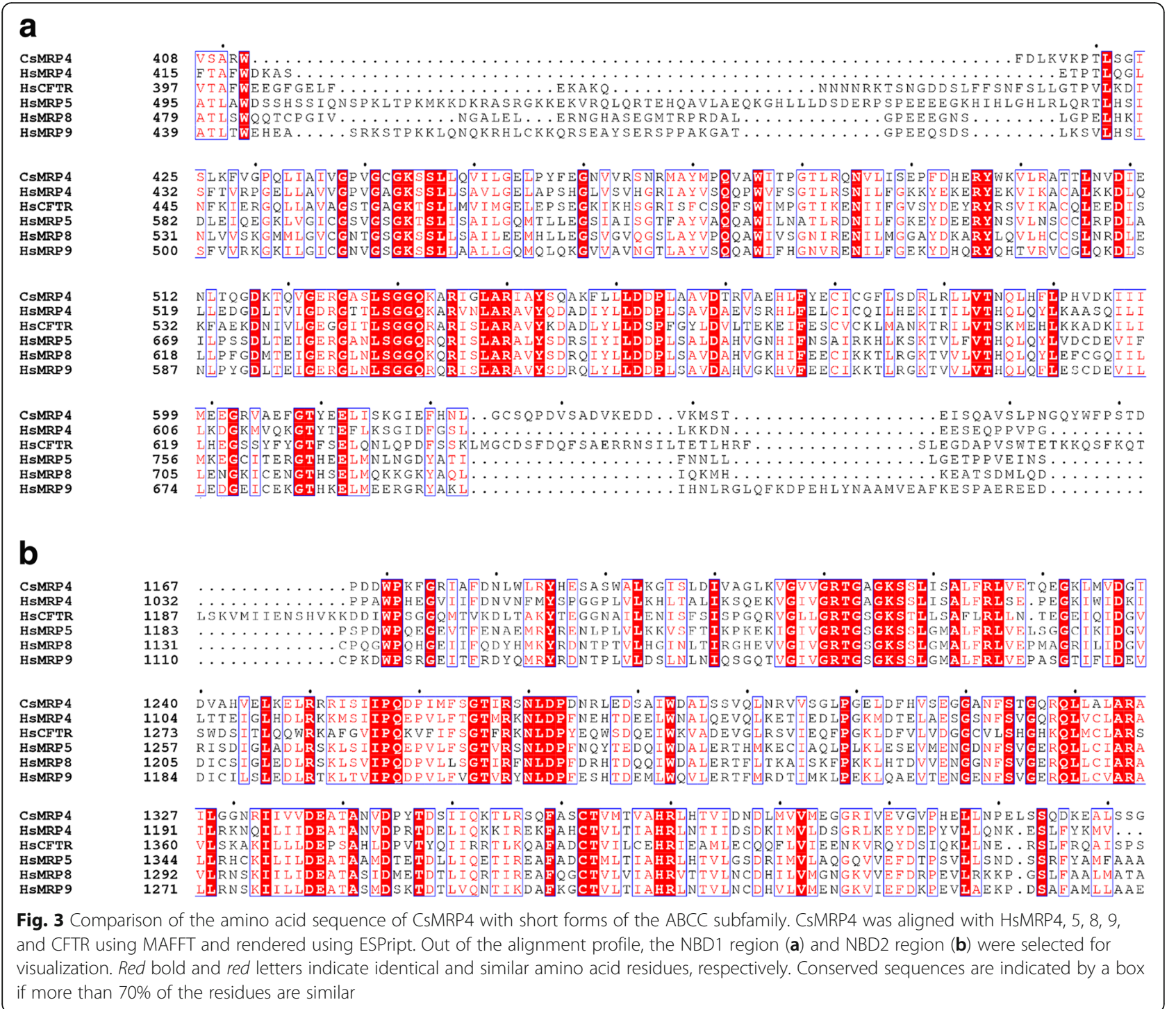

\section{Docking simulation for bile acid binding}

Various substrates were moved out via binding with MRP4 transporters. The export of inhibitors and bile acids can confer drug resistance and bile recirculation, respectively. Three MRP1 proteins and a P-gp, used as the four PDB templates, showed an open inward-facing conformation with inner cavity, which appears to be suitable for substrate uptake. These templates provided a possible structural foundation to perform in silico prediction of ligand binding by docking simulation. Thus, we investigated ligands and their binding sites for CsMRP4 using two methods. First, probable ligands were analyzed based on the identification of analogs with similar binding sites as the solved structures using $\mathrm{COACH}$ [28]. Then, $\mathrm{Mg}^{2+}$ and ATP were predicted to bind to NBD1 and NBD2 of CsMRP4 based on data for HsMRP1 [41] (Table 1 and Fig. 2a). $\mathrm{Mg}^{2+}$ is necessary for ATP hydrolysis and results in the formation of Mg-ATP
Table 1 Ligand and ligand-binding residues predicted using $\mathrm{COACH}$

\begin{tabular}{llll}
\hline Ligand & Region & Consensus binding residues & PDB template \\
\hline ATP & NBD1 & W412, T420, V440, G441, C442, & 2CBZ_A \\
& & G443, K444, S445, S446, Q473 & \\
Mg $^{2+}$ & NBD1 & S445, Q473 & 2CBZ_A \\
AMP-PNP & NBD2 & $\begin{array}{l}\text { Y1185, A1192, T1212, G1213, A1214, } \\
\text { G1215, K1216, S1217, S1218, V1227, }\end{array}$ & \\
& & Q1258, E1338, H1369 \\
Mg ${ }^{2+}$ & NBD2 & S1217, Q1258, D1337, E1338, V1367 & 4FWI_B \\
2J8 & Cavity & L94, P98, M101, S348, L864, I1093, & 4M2T_A \\
& & V1097 & \\
QZ59-VAL Cavity & P98, I1093 & 4Q9J_A \\
0JZ & Cavity & Y349, L353, I1093, I1118, V1122 & 3G61_B \\
\hline
\end{tabular}

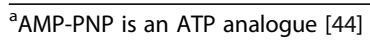


Table 2 Docking results between CsMRP4 and bile acids using AutoDock Vina

\begin{tabular}{|c|c|c|c|}
\hline Bile acids & PubChem ID & $\begin{array}{l}\text { Binding energy } \\
(\mathrm{kcal} / \mathrm{mol})\end{array}$ & $\begin{array}{l}\text { No. of } \\
\text { configurations }\end{array}$ \\
\hline $\begin{array}{l}\text { Taurolithocholic acid } \\
\left(\text { TLCA }{ }^{a}\right.\end{array}$ & SID 103579026 & -13.4 & 3 \\
\hline Lithocholic acid (LCA) & SID 103542513 & -12.2 & 3 \\
\hline $\begin{array}{l}\text { Taurochenodeoxycholic acid } \\
(T C D C A)^{a}\end{array}$ & SID 312642451 & -10.1 & 5 \\
\hline $\begin{array}{l}\text { Chenodeoxycholic acid } \\
\text { (CDCA) }\end{array}$ & SID 24875071 & -9.9 & 7 \\
\hline $\begin{array}{l}\text { Taurodeoxycholic acid } \\
\left(_{(T D C A)^{a}}\right.\end{array}$ & CID 2733768 & -9.7 & 5 \\
\hline Taurocholic acid (TCA) ${ }^{a}$ & SID 828139 & -9.3 & 6 \\
\hline Glycocholic acid (GCA) & SID 177011773 & -9.2 & 7 \\
\hline Deoxycholic acid (DCA) & CID 222528 & -8.4 & 5 \\
\hline Cholic acid (CA) ${ }^{\text {a }}$ & SID 223730521 & -8.1 & 5 \\
\hline
\end{tabular}

Binding affinities of bile acids to MRP4 in HEK cells [46]

dimers [42]. Moreover, the cyclic peptide inhibitor, QZ59-SSS (a.k.a. OZ-VAL or 2 J8), was predicted to bind to CsMRP4 according to the ligand-bound pockets of three experimentally characterized P-glycoproteins [43-45] (Table 1). Among them, Ile at the position 1093 was commonly involved in coordinating the inhibitor.

Secondly, docking simulations were performed using AutoDock Vina [33] to evaluate the binding energies of CsMRP4 with nine bile acids (Table 2 and Fig. 4). All the bile acids tested bound favorably to the inner cavity of CsMRP4 (Fig. 4a). Taurolithocholic acid (TLCA) (Fig. 4b) and LCA (Fig. 4c) showed the highest affinities with CsMRP4, whereas deoxycholic acid (DCA) (Fig. 4i) and cholic acid (CA) (Fig. 4j) revealed moderate affinities. Interestingly, our docking results are in line with previous transport assay data, which indicated that TLCA bound favorably to MRP4 at a low concentration, but other bile acids needed much higher concentrations [46]. TLCA had the highest affinity for MRP4 overexpressed in HEK cells, followed by taurochenodeoxycholic acid (TCDCA), taurodeoxycholic acid (TDCA), taurocholic acid (TCA), glycocholic acid (GCA) and cholic acid (CA). We also then added more primary and secondary bile acids such as LCA, chenodeoxycholic acid (CDCA), and DCA. Among them, LCA at 2-4 $\mu \mathrm{M}$ concentration was reported to have a significant adverse effect on the survival of juvenile $C$. sinensis [7]. Thus, the high affinity of LCA could be required for removing LCA from the worm's body for survival. However, these findings remain to be established at the biochemical level, which needs to be studied in the future.

\section{Developmental expression and tissue distribution}

CsMRP4 mRNA was expressed at both developmental stages, in the metacercariae and in the adults, but the expression in the metacercariae was 1.91 times higher (Fig. 5a). This result suggested that the metacercariae might need the transcript for the efflux of bile acids from the fluke's body during their survival in the bile

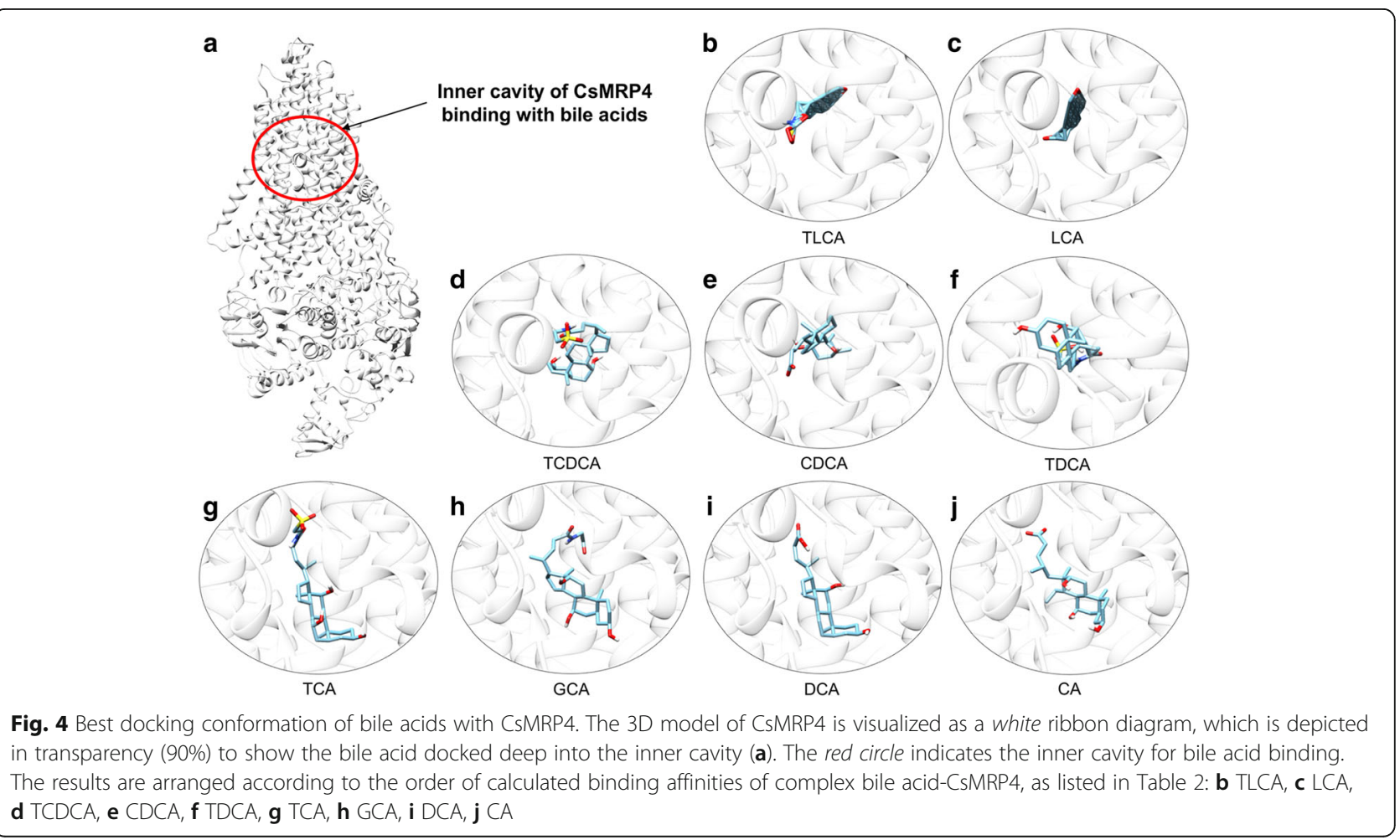



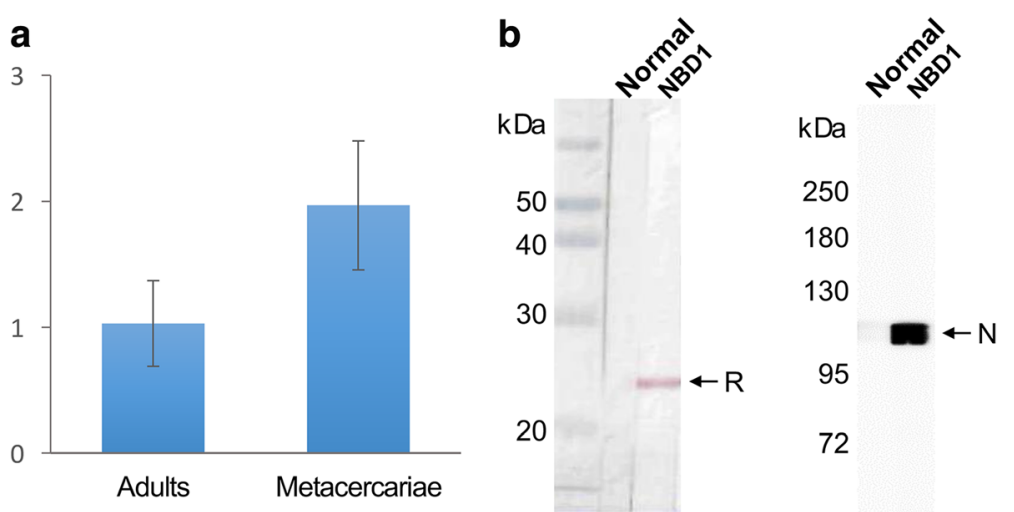

Fig. 5 Differential expression of the CSMRP4 and reactivity of mouse anti-NBD1 immune serum. a Relative mRNA level of CsMRP4 gene in the adults and metacercariae of $C$. sinensis, measured using Q-rt.-PCR. $\mathbf{b}$ Reaction detected by western blot and immuno-ECL. The mouse immune serum reacted well with rCsMRP4-NBD1 (left) and specifically detected native CsMRP4 (right) in the crude extracts of C. sinensis. Lane NBD1: mouse anti-NBD1 immune serum; Lane Normal: normal mouse serum; Abbreviations: R, recombinant protein; N, native CsMRP4

duct of the final host. In the metacercarial stage of $C$. sinensis, diverse genes have been reported to be highly expressed in response to environmentally induced changes, such as sodium/bile acid cotransporter and several heat-shock proteins [47]. Recently, the mRNA level of CsMRP7 was also reported to be elevated in the metacercariae [15].

The tissue distribution of CsMRP4 in both adults and metacercariae was investigated via immunohistochemistry. The NBD1 region was chosen as the immune antigen for mice immunization, since it was more specific than NBD2 and the non-membrane-spanning region. With this strategy, we successfully produced and purified NBD1 of ABCC subfamily transporters [15]. The 6x histidine-tagged rCsMRP4-NBD1 was then purified using Ni-NTA agarose (Additional file 8: Figure S5). The mouse anti-CsMRP4-NBD1 immune serum reacted well with rCsMRP4-NBD1 (24.9 kDa) by apparently detecting the native CsMRP4 in the crude extract of the adult worm (Fig. 5b). It was, therefore, applied to immunohistochemical staining. CsMRP4 was distributed mainly in the oral sucker and mesenchymal tissues of the adults (Fig. 6a, b) and metacercariae (Fig. 6c). Moreover, the ventral sucker of the metacercariae showed strong localization of CsMRP4.

CsMRP4 was mainly localized in the mesenchymal tissues in our study. In trematodes, several transporters have been found to be expressed in mesenchymal tissues. BSEP and MRP1 of adult Fasciola hepatica have been shown to be localized in not only mesenchymal tissues but also the tegumental cell layer, implying that the two transporters facilitate the diffusion of bile salts and chemicals in flukes [48]. CsMRP7, which might be involved in drug resistance, has also been found to be

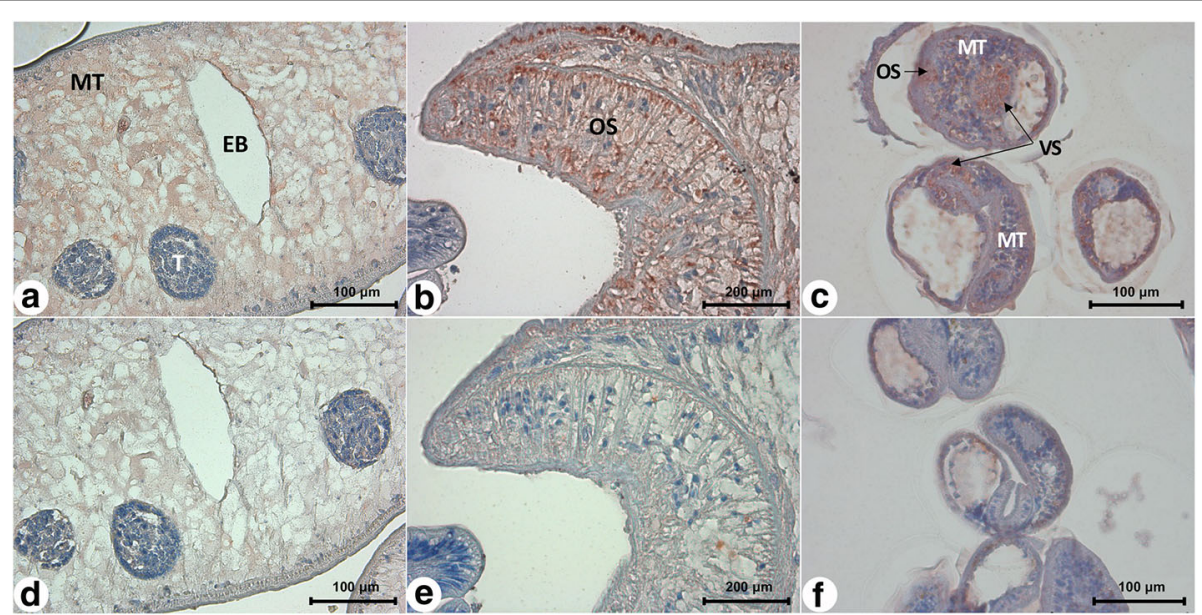

Fig. 6 Localization of CsMRP4 in C. sinensis adults $(\mathbf{a}, \mathbf{b}, \mathbf{d}, \mathbf{e})$ and metacercariae $(\mathbf{c}, \mathbf{f})$ detected by immunohistochemistry. Top panels $(\mathbf{a}-\mathbf{c})$ were stained with mouse anti-NBD1 immune serum and bottom panels (d-f) were stained with normal mouse serum. Abbreviations: EB, excretory bladder; MT, mesenchymal tissue; OS, oral sucker; T, testis; VS, ventral sucker 
expressed in the mesenchymal tissues [15]. It is therefore speculated that mesenchymal tissues may be regions of strategic importance for transporter functions throughout the body of flukes.

The function of CsMRP4 seems to be to export bile acids from the worm's body, which is similar to the function of typical MRP4. C. sinensis adults and metacercariae immerse themselves in bile juice, which can exert toxic effects and impair the tissues and cells of the worm' bodies. Bile acids have also been reported to decrease the locomotive cycles of juvenile F. hepatica and to provoke parasite death [49]. Together, these data suggest that $C$. sinensis needs to dilute the high concentrations of bile acids in the interior of the body by pumping them out and that CsMRP4 plays a role in transporting bile acids in coordination with other bile acid exporters.

\section{Conclusions}

In summary, we cloned and characterized CsMRP4 using computational, molecular, and biochemical approaches in this study. In addition to structural similarities, sequence similarities were also found between CsMRP4 and human MRP4 (39\% identity), and CsMRP4 was confirmed as belonging to the $\mathrm{ABCC}$ family. A reliable tertiary structure of CsMRP4 was also modeled and shown to have a common structural fold, MSD1-NBD1-MSD2-NBD2. When binding affinities of CsMRP4 with nine bile acids were tested through virtual docking simulation, the results indicated that CsMRP4 could be regarded as a bile transporter. The NBD2 of CsMRP4 was conserved more than NBD1, which was therefore used as a CsMRP4specific antigen for subsequent immunohistochemistry experiments. In the metacercariae and adults of $C$. sinensis, CsMRP4 was found to be mainly distributed in mesenchymal tissues, which suggested that these tissues are regions of strategic importance for transporter functions throughout the fluke's body. These findings suggest that CsMRP4 plays a role in exporting bile acids and inhibitors. The results from this study will also serve as a platform for further research on other bile transporters and homologues in flukes.

\section{Additional files}

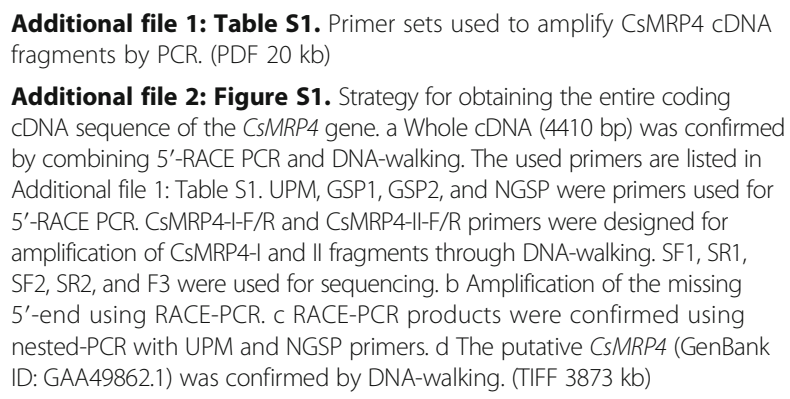

Additional file 1: Table S1. Primer sets used to amplify CsMRP4 CDNA fragments by PCR. (PDF $20 \mathrm{~kb}$ )

Additional file 2: Figure S1. Strategy for obtaining the entire coding CDNA sequence of the CSMRP4 gene. a Whole CDNA (4410 bp) was confirmed by combining 5'-RACE PCR and DNA-walking. The used primers are listed in Additional file 1: Table S1. UPM, GSP1, GSP2, and NGSP were primers used for 5'-RACE PCR. CSMRP4-I-F/R and CSMRP4-II-F/R primers were designed for amplification of CSMRP4-I and II fragments through DNA-walking. SF1, SR1, SF2, SR2, and F3 were used for sequencing. b Amplification of the missing 5'-end using RACE-PCR. C RACE-PCR products were confirmed using nested-PCR with UPM and NGSP primers. $d$ The putative CSMRP4 (GenBank ID: GAA49862.1) was confirmed by DNA-walking. (TIFF 3873 kb)

Additional file 3: Figure S2. The full cDNA coding sequence and deduced polypeptide sequence of CSMRP4. Through 5'-RACE and DNA-walking, the whole cDNA of $4410 \mathrm{bp}$ was verified to encode a polypeptide of 1496 aa. (TIFF 5203 kb)

Additional file 4: Table S2. Identities calculated between CSMRP4 and MRP/SUR/CFTR subfamily members. (PDF 29 kb)

Additional file 5: Figure S3. The residue-by-residue stereochemical quality of the CSMRP4 3D model. Ramachandran plot showed the residues in the most favored regions (90.7\%), additional allowed regions (8.4\%), generously allowed regions (0.6\%), and disallowed regions (0.3\%). Red (A, B, L), yellow (a, b, $\mathrm{I}, \mathrm{p})$, and light yellow $(\sim a, \sim b, \sim 1, \sim p)$ indicate the most favored regions, allowed regions, and generously allowed regions, respectively. White indicates disallowed regions. All the non-glycine and non-proline residues are shown as closed black squares, while glycines (non-end) are shown as closed black triangles. Disallowed residues are colored in red. (TIFF $993 \mathrm{~kb}$ )

Additional file 6: Figure S4. Accuracy of the non-bonded atomic contacts of the CSMRP4 3D model. The ERRAT plot shows the overall quality factor of $98.45 \%$. (TIFF $1786 \mathrm{~kb}$ )

Additional file 7: CsMRP4.pdb. The model of CsMRP4 was built using YASARA. (PDB $1780 \mathrm{~kb}$ )

Additional file 8: Figure S5. Amplification of CSMRP4-NBD1 (a) and purification of the recombinant protein (b). Abbreviations: M, molecular marker $(\mathrm{kDa})$; $\mathrm{U}$, uninduced total lysate; I, induced total lysate; $\mathrm{S}$, urea-treated clear supernatant; PT, Ni-NTA pass-through fraction; W, last washing; Elute 1-6, 1st to 6th fraction eluted from an Ni-NTA column. (TIFF $2938 \mathrm{~kb}$ )

\section{Abbreviations}

5'-RACE: 5'-rapid amplification of the CDNA ends; aa: Amino acid; ABC: ATP-binding cassette; BSEP: Bile salts export pump; CA: Cholic acid; CDCA: Chenodeoxycholic acid; CFTR: Cystic fibrosis transmembrane conductance regulator; CsMRP4: Multidrug resistance-associated protein 4; DCA: Deoxycholic acid; GCA: Glycocholic acid; LCA: Lithocholic acid; MSD: Membrane-spanning domain; NBD: Nucleotide-binding domain; P-gp: P-glycoprotein; Q-rtPCR: Quantitative real-time PCR; SUR: Sulfonylurea receptor; TCA: Taurocholic acid; TCDCA: Taurochenodeoxycholic acid; TDCA: Taurodeoxycholic acid;

TLCA: Taurolithocholic acid

\section{Acknowledgments}

Ok-Kyoung Lim, faculty from the Department of Pathology, Chung-Ang University, contributed the immunohistochemical samples of $C$. sinensis worms.

\section{Funding}

This research was supported by the National Research Foundation of Korea (NRF) funded by the Ministry of Science, ICT \& Future Planning (grant no. 2014R1A2A1A11051870).

\section{Availability of data and materials}

The data supporting the conclusions of this article are included within the article and its additional files.

\section{Authors' contributions}

SJH and WGY conceived and designed this project. JYL, FD, YL and WMS performed experiments. WGY and JHP analyzed data. WGY contributed to utilizing the analysis tools. FD, WGY and SJH wrote the paper. All authors read and approved the final manuscript.

Ethics approval and consent to participate

This study was carried out in strict accordance with the national guidelines outlined by Korean Laboratory Animal Act (No. KCDC-122-14-2A) of the Korean Centers for Disease Control and Prevention (KCDC). Animal handling and experimental procedures were reviewed and approved by the Institutional Animal Care and Use Committee at Chung-Ang University (Approval Number 2015-00005).

\section{Consent for publication}

Not applicable.

\section{Competing interests}

The authors declare that they have no competing interests. 


\section{Publisher's Note}

Springer Nature remains neutral with regard to jurisdictional claims in published maps and institutional affiliations.

\section{Author details}

'Department of Medical Environmental Biology, Chung-Ang University College of Medicine, Seoul 06974, South Korea. ${ }^{2}$ Department of Convergence Medicine, University of Ulsan College of Medicine and Asan Institute for Life Sciences, Asan Medical Center, Seoul 05505, South Korea. ${ }^{3}$ Department of Parasitology and Institute of Health Sciences, Gyeongsang National University School of Medicine, Jinju 52828, South Korea.

Received: 31 August 2017 Accepted: 5 November 2017 Published online: 21 November 2017

\section{References}

1. Yu SH, Kawanaka M, Li XM, Xu LQ, Lan CG, Rui L. Epidemiological investigation on Clonorchis sinensis in human population in an area of South China. Jpn J Infect Dis. 2003;56:168-71.

2. Rim HJ. Clonorchiasis: an update. J Helminthol. 2005:79:269-81.

3. Wang KX, Zhang RB, Cui YB, Tian Y, Cai R, Li CP. Clinical and epidemiological features of patients with clonorchiasis. World J Gastroenterol. 2004;10:446-8.

4. Keiser J, Utzinger J. Emerging foodborne trematodiasis. Emerg Infect Dis, 2005;11:1507-14

5. Bouvard V, Baan R, Straif K, Grosse Y, Secretan B, El Ghissassi F, et al. A review of human carcinogens - part B: biological agents. Lancet Oncol. 2009;10:321-2.

6. Hofmann AF. The continuing importance of bile acids in liver and intestinal disease. Arch Intern Med. 1999;159:2647-58.

7. Li S, Kim TI, Yoo WG, Cho PY, Kim TS, Hong SJ. Bile components and amino acids affect survival of the newly excysted juvenile Clonorchis sinensis in maintaining media. Parasitol Res. 2008;103:1019-24.

8. Alrefai WA, Gill RK. Bile acid transporters: structure, function, regulation and pathophysiological implications. Pharm Res. 2007;24:1803-23.

9. Hopper E, Belinsky MG, Zeng H, Tosolini A, Testa JR, Kruh GD. Analysis of the structure and expression pattern of MRP7 (ABCC10), a new member of the MRP subfamily. Cancer Lett. 2001;162:181-91.

10. Slot AJ, Molinski SV, Cole SPC. Mammalian multidrug-resistance proteins (MRPs). Essays Biochem. 2011;50:179-207.

11. Rius M, Hummel-Eisenbeiss J, Hofmann AF, Keppler D. Substrate specificity of human ABCC4 (MRP4)-mediated cotransport of bile acids and reduced glutathione. Am J Physiol Gastrointest Liver Physiol. 2006;290:G640-9.

12. Leggas M, Adachi M, Scheffer GL, Sun D, Wielinga P, Du G, et al. Mrp4 confers resistance to topotecan and protects the brain from chemotherapy. Mol Cell Biol. 2004;24:7612-21.

13. Roberts LM, Black DS, Raman C, Woodford K, Zhou M, Haggerty JE, et al. Subcellular localization of transporters along the rat blood-brain barrier and blood-cerebral-spinal fluid barrier by in vivo biotinylation. Neuroscience. 2008:155:423-38

14. Zhang Y-K, Wang Y-J, Gupta P, Chen Z-S. Multidrug resistance proteins (MRPS) and cancer therapy. AAPS J. 2015;17:802-12.

15. Dai F, Yoo WG, Lee JY, Lu Y, Pak JH, Sohn WM, et al. Molecular and structural characteristics of multidrug resistance-associated protein 7 in Chinese liver fluke Clonorchis sinensis. Parasitol Res. 2017;116:953-62.

16. UniProt C. UniProt: a hub for protein information. Nucleic Acids Res. 2015; 43:D204-12.

17. Marchler-Bauer A, Bo Y, Han L, He J, Lanczycki CJ, Lu S, et al. CDD/SPARCLE: functional classification of proteins via subfamily domain architectures. Nucleic Acids Res. 2017;45:D200-D3.

18. Jones P, Binns D, Chang HY, Fraser M, Li W, McAnulla C, et al. InterProScan 5: genome-scale protein function classification. Bioinformatics. 2014;30: 1236-40.

19. Katoh K, Standley DM. MAFFT multiple sequence alignment software version 7: improvements in performance and usability. Mol Biol Evol. 2013; $30: 772-80$

20. Tamura K, Stecher G, Peterson D, Filipski A, Kumar S. MEGA6: Molecular Evolutionary Genetics Analysis version 6.0. Mol Biol Evol. 2013;30:2725-9.

21. Krieger E, Nabuurs SB, Vriend G. Homology modeling. Methods Biochem Anal. 2003;44:509-23.
22. Altschul SF, Madden TL, Schaffer AA, Zhang J, Zhang Z, Miller W, et al. Gapped BLAST and PSI-BLAST: a new generation of protein database search programs. Nucleic Acids Res. 1997;25:3389-402.

23. Bernstein FC, Koetzle TF, Williams GJ, Meyer EF Jr, Brice MD, Rodgers JR, et al. The protein data bank. A computer-based archival file for macromolecular structures. Eur J Biochem. 1977;80:319-24.

24. Xu D, Zhang Y. Improving the physical realism and structural accuracy of protein models by a two-step atomic-level energy minimization. Biophys J. 2011;101:2525-34.

25. Lovell SC, Davis IW, Arendall WB 3rd, de Bakker PI, Word JM, Prisant MG, et al. Structure validation by Calpha geometry: phi, psi and Cbeta deviation. Proteins. 2003;50:437-50.

26. Colovos C, Yeates TO. Verification of protein structures: patterns of nonbonded atomic interactions. Protein Sci. 1993;2:1511-9.

27. Gouet $P$, Robert $X$, Courcelle E. ESPript/ENDscript: extracting and rendering sequence and 3D information from atomic structures of proteins. Nucleic Acids Res. 2003:31:3320-3.

28. Yang J, Roy A, Zhang Y. Protein-ligand binding site recognition using complementary binding-specific substructure comparison and sequence profile alignment. Bioinformatics. 2013;29:2588-95.

29. Kim S, Thiessen PA, Bolton EE, Chen J, Fu G, Gindulyte A, et al. PubChem substance and compound databases. Nucleic Acids Res. 2016;44:D1202-13.

30. O'Boyle NM, Morley C, Hutchison GR. Pybel: a python wrapper for the OpenBabel cheminformatics toolkit. Chem Cent J. 2008;2:5.

31. Dallakyan S, Olson AJ. Small-molecule library screening by docking with PyRx. Methods Mol Biol. 2015;1263:243-50.

32. Marzaro G, Ferrarese A, Chilin A. Autogrid-based clustering of kinases: selection of representative conformations for docking purposes. Mol Divers. 2014; 18:611-9.

33. Trott O, Olson AJ. AutoDock Vina: improving the speed and accuracy of docking with a new scoring function, efficient optimization, and multithreading. J Comput Chem. 2010;31:455-61.

34. Huang CC, Meng EC, Morris JH, Pettersen EF, Ferrin TE. Enhancing UCSF Chimera through web services. Nucleic Acids Res. 2014;42:W478-84.

35. Yoo WG, Kim TI, Li S, Kwon OS, Cho PY, Kim TS, et al. Reference genes for quantitative analysis on Clonorchis sinensis gene expression by real-time PCR. Parasitol Res. 2009;104:321-8.

36. Livak KJ, Schmittgen TD. Analysis of relative gene expression data using real-time quantitative PCR and the 2(-Delta Delta C(T)) method. Methods. 2001;25:402-8.

37. Bai X, Lee JY, Kim TI, Dai F, Lee TJ, Hong SJ. Molecular cloning and characterization of growth factor receptor bound-protein in Clonorchis sinensis. PLoS One. 2014:9:e85577.

38. ter Beek J, Guskov A, Slotboom DJ. Structural diversity of ABC transporters. J Gen Physiol. 2014;143:419-35.

39. Locher KP. Mechanistic diversity in ATP-binding cassette (ABC) transporters. Nat Struct Mol Biol. 2016:23:487-93.

40. Laskowski RA, Rullmannn JA, MacArthur MW, Kaptein R, Thornton JM. AQUA and PROCHECK-NMR: programs for checking the quality of protein structures solved by NMR. J Biomol NMR. 1996;8:477-86.

41. Ramaen O, Leulliot N, Sizun C, Ulryck N, Pamlard O, Lallemand JY, et al. Structure of the human multidrug resistance protein 1 nucleotide binding domain 1 bound to Mg2+/ATP reveals a non-productive catalytic site. J Mol Biol. 2006;359:940-9.

42. Chaves LA, Gadsby DC. Cysteine accessibility probes timing and extent of NBD separation along the dimer interface in gating CFTR channels. J Gen Physiol. 2015;145:261-83.

43. Aller SG, Yu J, Ward A, Weng Y, Chittaboina S, Zhuo R, et al. Structure of P-glycoprotein reveals a molecular basis for poly-specific drug binding. Science 2009:323:1718-22

44. Li J, Jaimes KF, Aller SG. Refined structures of mouse P-glycoprotein. Protein Sci. 2014:23:34-46.

45. Szewczyk P, Tao H, McGrath AP, Villaluz M, Rees SD, Lee SC, et al. Snapshots of ligand entry, malleable binding and induced helical movement in P-glycoprotein. Acta Crystallogr D Biol Crystallogr. 2015;71:732-41.

46. Zelcer N, Reid G, Wielinga P, Kuil A, van der Heijden I, Schuetz JD, et al. Steroid and bile acid conjugates are substrates of human multidrug-resistance protein (MRP) 4 (ATP-binding cassette C4). Biochem J. 2003:371:361-7.

47. Yoo WG, Kim DW, Ju JW, Cho PY, Kim TI, Cho SH, et al. Developmental transcriptomic features of the carcinogenic liver fluke, Clonorchis sinensis. PLoS Negl Trop Dis. 2011;e1208:5. 
48. Kumkate $\mathrm{S}$, Chunchob S, Janvilisri T. Expression of ATP-binding cassette multidrug transporters in the giant liver fluke Fasciola gigantica and their possible involvement in the transport of bile salts and anthelmintics. Mol Cell Biochem. 2008:317:77-84.

49. Sukhdeo MV, Keith S, Mettrick DF. The effects of bile on the locomotory cycle of Fasciola hepatica. J Parasitol. 1988;74:493-5.

Submit your next manuscript to BioMed Central and we will help you at every step:

- We accept pre-submission inquiries

- Our selector tool helps you to find the most relevant journal

- We provide round the clock customer support

- Convenient online submission

- Thorough peer review

- Inclusion in PubMed and all major indexing services

- Maximum visibility for your research

Submit your manuscript at www.biomedcentral.com/submit 